\title{
TOLERANCE OF AUTOCHTHONOUS LACTIC ACID BACTERIA TO DIFFERENT PROCESSING CONDITIONS IN VITRO
}

\author{
Mirjana Ž. Grujović*, Katarina G. Mladenović, Ljiljana R. Čomić \\ University of Kragujevac, Faculty of Science, Department of Biology and Ecology, Radoja Domanovića 12, \\ 34000 Kragujevac, Serbia
}

\begin{abstract}
In this paper, the effect of different temperatures, $\mathrm{pH}$, and $\mathrm{NaCl}$ concentration on the growth of autochthonous lactic acid bacteria isolated from traditionally made Serbian cheese (Sokobanja area) was investigated by using the spectrophotometric method. Growth of tested Lactobacillus (Lb. fermentum, Lb. plantarum, and Lb. brevis) and Lactococcus lactis subsp. lactis biovar. diacetylactis five isolates were better in acidic $\mathrm{pH}$, while the growth of Enterococcus isolates (E. durans, E. faecium, and E. faecalis) was better in basic pH, at $37{ }^{\circ} \mathrm{C}$. At $4{ }^{\circ} \mathrm{C}$ after $24 \mathrm{~h}$, none of the tested bacteria showed growth. Since the autochthonous isolates were tolerant to a tested range of dairy processing conditions, further studies need to include the characterization of enzymatic activity of selected isolates, as well as the ability to use these isolates like starter cultures or food supplements in dairy or non-dairy products.
\end{abstract}

Key words: lactic acid bacteria, cheese microbiology, processing conditions, growth ability

\section{INTRODUCTION}

Southeastern Serbia is a specific geographical area, which had a long-time tradition of specific manufacturing artisanal dairy products. In this region, inhabitants produce dairy products by spontaneous milk fermentation, without the addition of bacterial starter cultures. The products made in this way represent a source of autochthonous bacterial strains that could have probiotic potential and serve as natural starter cultures. One of the traditionally made fermented products from this area is Sokobanja cheese, made in Southeastern Serbia (Sokobanja area) (Uroić et al., 2014; Muruzović, Mladenović, Žugić Petrović \& Čomić, 2018a).

In the process of cheese making, bacterial starter culture needs to be tolerant to a variety of processing conditions, including differences in temperature, salinity, and acid environment
(Rao, Pintado, Stevens \& Guyot, 2004; Gutiérrez-Méndez et al., 2010). Also, the tolerance to the gastrointestinal conditions is one of the major requirements for auto-chthonous bacteria to be considered as probiotics (Hernandez-Hernandez et al., 2012). Ibourahema, Dauphin, Jacqueline \& Thonart (2008) indicated that the capability of lactic acid bacteria (LAB) to grow at high temperatures is a desirable characteristic because a high fermentation temperature decreases contamination by other microorganisms. Several studies showed that temperature and $\mathrm{pH}$ have a great influence on the growth of $\mathrm{LAB}$ and that optimal $\mathrm{pH}$ for the growth of LAB was in the range from 6.3 to 6.9 (Adamberg, Kask, Laht \& Paalme, 2003; Muruzović, Mladenović \& Čomić, 2018b). According to Fontana, Bermudez-Brito, Plaza- 
Diaz, Muñoz-Quezada \& Gil (2013), isolates of Lactobacillus spp. showed tolerance to low $\mathrm{pH}$. Menconi et al. (2014) indicated that LABs were tolerant to $\mathrm{pH} 3.0$ and in the presence of $6.5 \%$ of $\mathrm{NaCl}$. According to Mohd Adnan \& Tan (2007), high osmosis tolerance is a desirable characteristic of LABs to be used as commercial strains in the dairy industry.

The isolates tested in this study present a part of the unexplored microflora of cheese from Serbia. Since the cheese is produced from raw cow's milk in local households, this study aimed to optimize some processing conditions (different temperatures, $\mathrm{pH}$, and $\mathrm{NaCl}$ concentrations) for the growth of isolated LAB. Also, the aim was to examine in which combination of factors their growth is higher.

\section{MATERIALS AND METHODS}

\section{LAB isolates}

The effects of different temperature, $\mathrm{pH}$, and salt concentration $(\mathrm{NaCl})$ were tested against 7 species of autochthonous lactic acid bacteria (13 isolates) isolated from Sokobanja cheese (Southeastern Serbia): Enterococcus durans KGPMF10, E. faecium KGPMF14, E. faecalis KGPMF47, E. faecalis KGPMF48, E. faecalis KGPMF49, Lactobacillus fermentum KGPMF28, Lb. brevis KGPMF35, Lb. plantarum KGPMF62, Lactococcus lactis subsp. lactis biovar. diacetylactis KGPMF50, L. lactis subsp. lactis biovar. diacetylactis KGPMF54, $L$. lactis subsp. lactis biovar. Diacetylactis KGPMF55, L. lactis subsp. lactis biovar. diacetylactis KGPMF57, L. lactis subsp. lactis biovar. diacetylactis KGPMF59. These isolates were identified by using API $50 \mathrm{CH}$ and Microgen Strep ID tapes for preliminary identification. The final identification was done by using a MALDI-TOF mass spectrophotometry (Muruzović, Mladenović, Žugić Petrović \& Čomić, 2018a; Muruzović, Mladenović, Đilas, Stefanović \& Čomić, 2018c; Grujović, Mladenović, Žugić Petrović \& Čomić, 2019). E. faecalis ATCC 29211 (standard strain) and $L b$. plantarum LP 299v (commercial probiotic strain) were used for comparison of results. All isolates from Sokobanja cheese, as well as standard and probiotic strains were provided by the Microbiology Laboratory, Faculty of Science, University of Kragujevac, Serbia. The bacterial strains were kept in glycerol stock at -80 ${ }^{\circ} \mathrm{C}$ until their use.
Assay for determination of the effect of different temperatures, $\mathrm{pHs}$, and salt concentrations on the growth of LAB

Determination of the effect of different environmental conditions on the growth of bacteria was performed according to Thayer, Muller, Buchanan \& Phillips, (1987), with some modifications described in detail in Muruzović, Mladenović \& Čomić (2018b). The growth was measured at a spectrophotometer at $600 \mathrm{~nm}$.

The effect of different temperatures on the growth of LAB was examined as follows: in 1 $\mathrm{ml}$ of MRS broth (Torlak, Belgrade, Serbia), it was added $10 \mu \mathrm{l}$ of initial bacterial suspension (contained $10^{8}$ colony-forming units $\mathrm{CFU} / \mathrm{ml}$ ). The turbidity of the initial suspension was adjusted using a 0.5 McFarland densitometer (Biosan, Latvia). Three samples were prepared, each for one tested temperature $\left(4{ }^{\circ} \mathrm{C}, 20{ }^{\circ} \mathrm{C}\right.$, and $37{ }^{\circ} \mathrm{C}$ ). These temperatures were selected because the temperature at $4{ }^{\circ} \mathrm{C}$ is a temperature of cheese storage in the refrigerator, the temperature of Sokobanja cheese making and preservation in the household is at $20{ }^{\circ} \mathrm{C}$ and the temperature for optimal bacterial growth in most cases is at $37^{\circ} \mathrm{C}$. Samples were incubated for $24 \mathrm{~h}$. Each experiment was performed in triplicate. Sterility control was uninoculated MRS broth.

The effect of different $\mathrm{pH}$ in different temperatures was examined in modified MRS broths. By adding a concentrated $\mathrm{HCl}$ (Zorka Pharma, Šabac, Serbia), it was obtained acidic media (pH 5.5) and with adding a 30\% $\mathrm{NaOH}$ (Zorka Pharma, Šabac, Serbia), it was obtained neutral

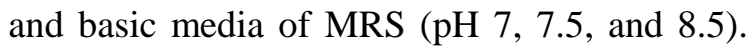
Growth control was at $\mathrm{pH} 6.5$ (pH of pure MRS broth). In $1 \mathrm{ml}$ of each type of modified media, it was added $10 \mu \mathrm{l}$ of the initial bacterial suspension. Samples were incubated at $4{ }^{\circ} \mathrm{C}, 20$ ${ }^{\circ} \mathrm{C}$, and $37{ }^{\circ} \mathrm{C}$ for $24 \mathrm{~h}$. Each experiment was performed in triplicate. Sterility controls were uninoculated modified MRS broths, prepared in every tested $\mathrm{pH}$ value. To examine the effect of different $\mathrm{NaCl}$ concentrations on the growth of tested LAB, MRS broths were prepared, modified with the addition of different concentrations of $\mathrm{NaCl}$ (Zorka Pharma, Šabac, Serbia) $(\mathrm{w} / \mathrm{v})(4 \%, 6.5 \%, 8 \%)$. In $1 \mathrm{ml}$ of modified media, it was added $10 \mu \mathrm{l}$ of initial bacterial suspension (contained $10^{8}$ colony-forming units $\mathrm{CFU} / \mathrm{ml}$ ). Samples were incubated at $4{ }^{\circ} \mathrm{C}, 20$ ${ }^{\circ} \mathrm{C}$, and $37{ }^{\circ} \mathrm{C}$ for $24 \mathrm{~h}$. Each experiment was 
performed in triplicate. Sterility controls were uninoculated modified MRS broths, prepared in each tested salt concentration.

\section{Statistical analysis}

All data were presented as means \pm standard deviations using Microsoft Excel (Redmond, Washington, DC, USA). Paired - Samples Ttest was used to compare the influence of temperatures on the growth of each particular isolate. Data were analyzed using SPSS version 20 software (SPSS Inc., Chicago, IL, USA).

\section{RESULTS AND DISCUSSION}

In this paper, it was investigated the effect of some dairy processing conditions (different temperatures, $\mathrm{pH}$, and $\mathrm{NaCl}$ concentrations) on the growth of LABs, previously isolated from traditionally made Sokobanja cheese (Southeastern Serbia). LABs were incubated in standard and modified MRS media, at three different temperatures $\left(4{ }^{\circ} \mathrm{C}, 20^{\circ} \mathrm{C}, 37^{\circ} \mathrm{C}\right)$, for $24 \mathrm{~h}$. After incubation, it was noticed that there was no growth at $4{ }^{\circ} \mathrm{C}$. The rest of the results are presented below, in Tables 1 - 4 .

Tested LABs showed better growth in acidic media, at $37{ }^{\circ} \mathrm{C}$, compared with growth at $20^{\circ} \mathrm{C}$ $(\mathrm{P}<0.05)$. Lactobacillus and Lactococcus isolates showed the growth ability at both tested temperatures. Besides, growth at $37{ }^{\circ} \mathrm{C}$ was better. Further, it was noticed that, at $20{ }^{\circ} \mathrm{C}$, growth was better at $\mathrm{pH} 7$ than in growth control (pH 6.5) (Table 1).

Tested Enterococcus isolates showed the growth ability in all tested $\mathrm{pH}$, at both tested temperatures (Table 2), but their growth was better in basic $\mathrm{pH}$. Based on the results, it could be concluded that the temperature had a greater impact, compared to the $\mathrm{pH}(\mathrm{P}<0.05)$.

Different concentrations of $\mathrm{NaCl}$ showed an inhibitory effect on the growth of tested isolates of LABs (Tables 3 and 4). The growth of all isolates was reduced in the presence of $4 \%$ of $\mathrm{NaCl}$, at both tested temperatures. Different $\mathrm{NaCl}$ concentrations showed a significant influence on bacterial growth at $20{ }^{\circ} \mathrm{C}$ when compared to the same concentrations at $37{ }^{\circ} \mathrm{C}$ ( $\mathrm{P}<0.05)$. Tested Lactobacillus and Lactococcus isolates showed a tolerance up to $6.5 \%$ of $\mathrm{NaCl}$ (Table 3), while tested Enterococcus isolates showed tolerance to the presence of $\mathrm{NaCl}$ up to $8 \%$, especially at $37^{\circ} \mathrm{C}$ (Table 4).
The process of cheese ripening is very complex, in which the typical cheese characteristics (flavor and texture) are formed by the action of numerous enzymes derived from the milk, the rennet, the starter culture bacteria, and the nonstarter bacteria (Fox, Guinee, Cogan \& Mcsweeney, 2017). Most of the methods acelerated to cheese ripening is focused on enhancing proteolysis and lipolysis by for instance ripening at elevated temperatures, the addition of enzymes (e.g. lipases, proteinases, and peptidases), using a well-known starter culture, associated culture and genetically modified cultures (Van Mastrigt, Gallegos Tejeda, Kristensen, Abee \& Smid, 2018). Van Mastrigt, Gallegos Tejeda, Kristen-sen, Abee \& Smid (2018) also indicated that it is interesting to study aroma formation outside the cheese matrix, from a scientific and a technological perspective because such studies could help to optimize or steer to aroma formation by LAB applied as food supplements in dairy or dairylike products. It is important that, in the process of Sokobanja cheese making, it was not added any bacterial starter culture. A liquid rennet of microbiological origin based on chymosin obtained from the fungi Rhizomucor miehei and Mucor miehei was used for milk coagulation (Muruzović, Mladenović, Žugić Petrović \& Čomić, 2018a). Therefore, it could be assumed that tested isolates of LAB present a part of the autochthonous community of cheese, originated from raw milk and the endogenous culture. According to the results described in Muruzović, Mladenović, Žugić Petrović \& Čomić, (2018a) and Muruzović, Đilas, Stefanović \& Čomić (2018c) the chese samples were three days old and it belongs to the group of full-fat, acid-curd soft cheese groups. In the process of cheese production, the cheese was salted (6-8\%), based on the total weight of the cheese (Muruzović, Mladenović, Žugić Petrović \& Čomić, 2018a; Muruzović, Mladenović, Đilas, Stefanović \& Čomić, 2018c). These results were the first reason why we tested the influence of the chosen $\mathrm{pH}$ and $\mathrm{NaCl}$ concentrations on the growth of selected bac-teria. Also, it is well-known that the tolerance to $\mathrm{pH}$ is one of the major criteria for $\mathrm{LAB}$ to be considered as probiotics and the tolerance to different processing conditions is important for the possible use of LABs as starter cultures in the dairy industry (Zago et al., 2011; Kavitha \& Devasena, 2013). 
Table 1.

The effect of different $\mathrm{pH}$ and temperatures on the growth of isolated Lactobacillus spp. and Lactococcus spp.

\begin{tabular}{|c|c|c|c|c|c|c|c|c|c|c|c|}
\hline & \multirow{2}{*}{$\begin{array}{r}\mathbf{p H} \\
\text { Temperature }\end{array}$} & \multicolumn{2}{|c|}{5.5} & \multicolumn{2}{|c|}{$6.5^{*}$} & \multicolumn{2}{|c|}{7.0} & \multicolumn{2}{|c|}{7.5} & \multicolumn{2}{|c|}{8.5} \\
\hline & & $20{ }^{\circ} \mathrm{C}$ & $37^{\circ} \mathrm{C}$ & $20^{\circ} \mathrm{C}$ & $37^{\circ} \mathrm{C}$ & $20{ }^{\circ} \mathrm{C}$ & $37^{\circ} \mathrm{C}$ & $20^{\circ} \mathrm{C}$ & $37^{\circ} \mathrm{C}$ & $20{ }^{\circ} \mathrm{C}$ & $37^{\circ} \mathrm{C}$ \\
\hline Species & Isolate & & & & & & & & & & \\
\hline $\begin{array}{l}\text { Lb. } \\
\text { fermentum }\end{array}$ & KGPMF28 & $\begin{array}{c}0.22 \pm 0.00^{\mathrm{a}} \\
\quad(63.67)\end{array}$ & $\begin{array}{c}1.70 \pm 0.04^{\mathrm{b}} \\
(97.7)\end{array}$ & $0.33 \pm 0.00^{\mathrm{c}}$ & $1.74 \pm 0.08^{b}$ & $\begin{array}{c}0.41 \pm 0.01^{\mathrm{d}} \\
(124.24)\end{array}$ & $\begin{array}{c}1.93 \pm 0.02^{\mathrm{e}} \\
(110.92)\end{array}$ & $\begin{array}{c}0.36 \pm 0.02^{\mathrm{f}} \\
(109.09)\end{array}$ & $\begin{array}{c}0.80 \pm 0.01^{\mathrm{g}} \\
(45.98)\end{array}$ & $\begin{array}{c}0.04 \pm 0.00^{\mathrm{h}} \\
\quad(12.12)\end{array}$ & $\begin{array}{c}0.65 \pm 0.05^{\mathrm{i}} \\
\quad(37.36)\end{array}$ \\
\hline Lb. brevis & KGPMF35 & $\begin{array}{c}0.23 \pm 0.01^{\mathrm{a}} \\
(53.49)\end{array}$ & $\begin{array}{c}1.98 \pm 0.02^{b} \\
(95.65)\end{array}$ & $0.43 \pm 0.02^{\mathrm{c}}$ & $2.07 \pm 0.03^{\mathrm{d}}$ & $\begin{array}{c}0.49 \pm 0.02^{\mathrm{e}} \\
(113.95)\end{array}$ & $\begin{array}{l}2.05 \pm 0.02^{\mathrm{d}} \\
(99.03)\end{array}$ & $\begin{array}{c}0.38 \pm 0.00^{\mathrm{e}} \\
(88.37)\end{array}$ & $\begin{array}{c}1.90 \pm 0.00^{f} \\
(91.79)\end{array}$ & $\begin{array}{c}0.17 \pm 0.00^{g} \\
(39.53)\end{array}$ & $\begin{array}{c}1.66 \pm 0.02^{\mathrm{h}} \\
(80.19)\end{array}$ \\
\hline \multirow{2}{*}{$\begin{array}{l}\text { Lb. } \\
\text { plantarum }\end{array}$} & KGPMF62 & $\begin{array}{c}0.13 \pm 0.03^{\mathrm{a}} \\
(29.55)\end{array}$ & $\begin{array}{c}1.98 \pm 0.02^{b} \\
(97.06)\end{array}$ & $0.44 \pm 0.03^{\mathrm{c}}$ & $2.04 \pm 0.00^{\mathrm{d}}$ & $\begin{array}{c}0.40 \pm 0.02^{\mathrm{c}} \\
(90.91)\end{array}$ & $\begin{array}{c}2.00 \pm 0.03^{\mathrm{d}} \\
(98.04)\end{array}$ & $\begin{array}{c}0.28 \pm 0.00^{\mathrm{e}} \\
(63.64)\end{array}$ & $\begin{array}{c}1.83 \pm 0.02^{f} \\
(89.71)\end{array}$ & $\begin{array}{c}0.11 \pm 0.00^{\mathrm{g}, \mathrm{a}} \\
(25)\end{array}$ & $\begin{array}{c}1.69 \pm 0.03^{\mathrm{h}} \\
(82.84)\end{array}$ \\
\hline & LP 299v & $\begin{array}{c}0.25 \pm 0.00^{\mathrm{a}} \\
(86.21)\end{array}$ & $\begin{array}{c}0.62 \pm 0.02^{\mathrm{b}} \\
(126.53)\end{array}$ & $0.29 \pm 0.02^{\mathrm{c}}$ & $0.49 \pm 0.02^{\mathrm{d}}$ & $\begin{array}{c}0.30 \pm 0.00^{\mathrm{c}} \\
(103.45)\end{array}$ & $\begin{array}{c}0.70 \pm 0.01^{\mathrm{e}} \\
(142.86)\end{array}$ & $\begin{array}{c}0.30 \pm 0.00^{\mathrm{c}} \\
(103.45)\end{array}$ & $\begin{array}{c}0.67 \pm 0.01^{\mathrm{e}} \\
(136.74)\end{array}$ & $\begin{array}{c}0.01 \pm 0.00^{\mathrm{f}} \\
(3.15)\end{array}$ & $\begin{array}{c}0.09 \pm 0.02^{\mathrm{g}} \\
(18.37)\end{array}$ \\
\hline \multirow{5}{*}{$\begin{array}{l}\text { L. lactis } \\
\text { subsp. lactis } \\
\text { biovar. } \\
\text { diacetylactis }\end{array}$} & KGPMF50 & $\begin{array}{c}0.09 \pm 0.00^{\mathrm{a}} \\
(39.13)\end{array}$ & $\begin{array}{c}1.39 \pm 0.28^{b} \\
(118.80)\end{array}$ & $0.23 \pm 0.02^{\mathrm{c}}$ & $1.17 \pm 0.01^{\mathrm{d}}$ & $\begin{array}{c}0.24 \pm 0.00^{\mathrm{c}} \\
(104.35)\end{array}$ & $\begin{array}{c}1.96 \pm 0.14^{\mathrm{e}} \\
(167.52)\end{array}$ & $\begin{array}{c}0.19 \pm 0.02^{f} \\
(82.61)\end{array}$ & $\begin{array}{c}1.68 \pm 0.02^{\mathrm{g}} \\
(143.59)\end{array}$ & $\begin{array}{l}0.14 \pm 0.01^{\mathrm{h}} \\
(60.87)\end{array}$ & $\begin{array}{c}0.64 \pm 0.02^{\mathrm{g}} \\
\quad(54.70)\end{array}$ \\
\hline & KGPMF54 & $\begin{array}{l}0.12 \pm 0.01^{\mathrm{a}} \\
\quad(70.59)\end{array}$ & $\begin{array}{c}2.03 \pm 0.02^{\mathrm{b}} \\
(100.50)\end{array}$ & $0.17 \pm 0.02^{\mathrm{c}}$ & $2.02 \pm 0.03^{b}$ & $\begin{array}{c}0.19 \pm 0.00^{\mathrm{c}} \\
(111.76)\end{array}$ & $\begin{array}{l}1.97 \pm 0.00^{\mathrm{d}} \\
(97.52)\end{array}$ & $\begin{array}{c}0.14 \pm 0.00^{\mathrm{e}, \mathrm{a}} \\
(82.35)\end{array}$ & $\begin{array}{c}1.86 \pm 0.00^{f} \\
(92.08)\end{array}$ & $\begin{array}{l}0.09 \pm 0.00^{\mathrm{g}} \\
\quad(52.94)\end{array}$ & $\begin{array}{c}1.69 \pm 0.03^{\mathrm{h}} \\
(83.67)\end{array}$ \\
\hline & KGPMF55 & $\begin{array}{l}0.06 \pm 0.02^{\mathrm{a}} \\
\quad(23.08)\end{array}$ & $\begin{array}{l}1.23 \pm 0.00^{\mathrm{b}} \\
(63.73)\end{array}$ & $0.26 \pm 0.02^{\mathrm{c}}$ & $1.93 \pm 0.01^{\mathrm{d}}$ & $\begin{array}{c}0.26 \pm 0.03^{c} \\
(100)\end{array}$ & $\begin{array}{l}1.79 \pm 0.35^{\mathrm{e}} \\
(92.75)\end{array}$ & $\begin{array}{l}0.23 \pm 0.02^{\mathrm{f}} \\
(88.46)\end{array}$ & $\begin{array}{c}1.76 \pm 0.01^{\mathrm{g}, \mathrm{e}} \\
(91.19)\end{array}$ & $\begin{array}{l}0.18 \pm 0.01^{\mathrm{h}} \\
\quad(69.23)\end{array}$ & $\begin{array}{c}1.30 \pm 0.08^{\mathrm{i}} \\
(67.36)\end{array}$ \\
\hline & KGPMF57 & $\begin{array}{l}0.21 \pm 0.02^{\mathrm{a}} \\
\quad(95.45)\end{array}$ & $\begin{array}{l}2.03 \pm 0.02^{\mathrm{b}} \\
\quad(99.51)\end{array}$ & $0.22 \pm 0.01^{\mathrm{a}}$ & $2.04 \pm 0.00^{\mathrm{b}}$ & $\begin{array}{l}0.19 \pm 0.00^{\mathrm{a}} \\
\quad(86.36)\end{array}$ & $\begin{array}{l}1.98 \pm 0.03^{\mathrm{c}} \\
(97.06)\end{array}$ & $\begin{array}{l}0.14 \pm 0.01^{\mathrm{d}} \\
\quad(63.64)\end{array}$ & $\begin{array}{c}1.87 \pm 0.02^{\mathrm{e}, \mathrm{c}} \\
\quad(91.67)\end{array}$ & $\begin{array}{c}0.11 \pm 0.00^{f} \\
(50)\end{array}$ & $\begin{array}{c}1.72 \pm 0.00^{\mathrm{g}} \\
(84.31)\end{array}$ \\
\hline & KGPMF59 & $\begin{array}{l}0.23 \pm 0.01^{\mathrm{a}} \\
\quad(88.46)\end{array}$ & $\begin{array}{c}1.98 \pm 0.02^{\mathrm{b}} \\
(97.06)\end{array}$ & $0.26 \pm 0.01^{\mathrm{a}}$ & $2.04 \pm 0.00^{\mathrm{c}}$ & $\begin{array}{l}0.25 \pm 0.02^{\mathrm{a}} \\
\quad(96.15)\end{array}$ & $\begin{array}{c}1.98 \pm 0.03^{\mathrm{d}, \mathrm{b}} \\
(97.06)\end{array}$ & $\begin{array}{c}0.24 \pm 0.00 \mathrm{a} \\
(92.31)\end{array}$ & $\begin{array}{c}1.86 \pm 0.00^{\mathrm{e}} \\
(91.18)\end{array}$ & $\begin{array}{c}0.12 \pm 0.00^{\mathrm{f}} \\
(46.15)\end{array}$ & $\begin{array}{c}1.71 \pm 0.03^{\mathrm{g}} \\
(83.82)\end{array}$ \\
\hline
\end{tabular}

Values are expressed as means \pm standard deviation measured on $600 \mathrm{~nm}$; the percent of growth was given in brackets;

Means in the two temperature columns or each particular isolate, with superscript with different letters, are significantly different at $P<0.05$;

${ }^{*}$ Growth control (100\% of growth) 
Table 2.

The effect of different $\mathrm{pH}$ and temperatures on the growth of isolated Enterococcus spp.

\begin{tabular}{|c|c|c|c|c|c|c|c|c|c|c|c|}
\hline & \multirow{2}{*}{$\begin{array}{r}\text { pH } \\
\text { Temperature }\end{array}$} & \multicolumn{2}{|c|}{5.5} & \multicolumn{2}{|c|}{$6.5^{*}$} & \multicolumn{2}{|c|}{7.0} & \multicolumn{2}{|c|}{7.5} & \multicolumn{2}{|c|}{8.5} \\
\hline & & $20^{\circ} \mathrm{C}$ & $37^{\circ} \mathrm{C}$ & $20^{\circ} \mathrm{C}$ & $37^{\circ} \mathrm{C}$ & $20^{\circ} \mathrm{C}$ & $37^{\circ} \mathrm{C}$ & $20^{\circ} \mathrm{C}$ & $37^{\circ} \mathrm{C}$ & $20^{\circ} \mathrm{C}$ & $37^{\circ} \mathrm{C}$ \\
\hline Species & Isolate & & & & & & & & & & \\
\hline $\begin{array}{l}\text { E. } \\
\text { durans }\end{array}$ & KGPMF10 & $\begin{array}{c}0.09 \pm 0.01^{\mathrm{a}} \\
\quad(14.06)\end{array}$ & $\begin{array}{c}1.16 \pm 0.01^{\mathrm{b}} \\
(68.64)\end{array}$ & $0.64 \pm 0.02^{\mathrm{c}}$ & $1.69 \pm 0.01^{\mathrm{d}}$ & $\begin{array}{l}0.63 \pm 0.00^{\mathrm{c}} \\
\quad(98.44)\end{array}$ & $\begin{array}{c}1.72 \pm 0.01^{\mathrm{d}} \\
(101.77)\end{array}$ & $\begin{array}{c}0.52 \pm 0.02^{\mathrm{e}} \\
\quad(81.25)\end{array}$ & $\begin{array}{c}1.81 \pm 0.01^{\mathrm{f}} \\
(107.10)\end{array}$ & $\begin{array}{c}0.44 \pm 0.02^{g} \\
\quad(68.75)\end{array}$ & $\begin{array}{c}1.65 \pm 0.02^{1} \\
\quad(97.63)\end{array}$ \\
\hline $\begin{array}{l}\text { E. } \\
\text { faecium }\end{array}$ & KGPMF14 & $\begin{array}{c}0.01 \pm 0.00^{\mathrm{a}} \\
(11.11)\end{array}$ & $\begin{array}{c}0.19 \pm 0.00^{\mathrm{b}} \\
(38.78)\end{array}$ & $0.09 \pm 0.01^{\mathrm{c}}$ & $0.49 \pm 0.00^{\mathrm{d}}$ & $\begin{array}{c}0.16 \pm 0.01^{\mathrm{e}} \\
(177.78)\end{array}$ & $\begin{array}{c}0.55 \pm 0.01^{f} \\
(112.24)\end{array}$ & $\begin{array}{c}0.12 \pm 0.00^{\mathrm{g}} \\
(133.33)\end{array}$ & $\begin{array}{c}0.69 \pm 0.00^{\mathrm{h}} \\
(140.81)\end{array}$ & $\begin{array}{c}0.11 \pm 0.00^{\mathrm{g}} \\
(122.22)\end{array}$ & $\begin{array}{c}0.76 \pm 0.00 \\
(161.23)\end{array}$ \\
\hline \multirow{4}{*}{$\begin{array}{l}\text { E. } \\
\text { faecalis }\end{array}$} & KGPMF47 & $\begin{array}{c}0.07 \pm 0.00^{\mathrm{a}} \\
(87.5)\end{array}$ & $\begin{array}{c}0.46 \pm 0.02^{\mathrm{b}} \\
(95.83)\end{array}$ & $0.08 \pm 0.01^{\mathrm{a}}$ & $0.48 \pm 0.01^{\mathrm{b}}$ & $\begin{array}{c}0.11 \pm 0.00^{\mathrm{a}} \\
(137.5)\end{array}$ & $\begin{array}{c}0.67 \pm 0.01^{\mathrm{c}} \\
(139.58)\end{array}$ & $\begin{array}{c}0.12 \pm 0.01^{\mathrm{a}} \\
(150)\end{array}$ & $\begin{array}{c}0.79 \pm 0.00^{\mathrm{d}} \\
(164.58)\end{array}$ & $\begin{array}{c}0.09 \pm 0.01^{\mathrm{a}} \\
(112.5)\end{array}$ & $\begin{array}{c}0.81 \pm 0.01 \\
(168.75)\end{array}$ \\
\hline & KGPMF48 & $\begin{array}{c}0.06 \pm 0.00^{\mathrm{a}} \\
\quad(50)\end{array}$ & $\begin{array}{c}0.28 \pm 0.03^{\mathrm{b}} \\
\quad(35.44)\end{array}$ & $0.12 \pm 0.02^{\mathrm{c}}$ & $0.79 \pm 0.01^{\mathrm{d}}$ & $\begin{array}{c}0.14 \pm 0.01^{\mathrm{c}} \\
(116.67)\end{array}$ & $\begin{array}{c}0.89 \pm 0.01^{\mathrm{e}} \\
\quad(112.66)\end{array}$ & $\begin{array}{c}0.16 \pm 0.01^{\mathrm{c}} \\
\quad(133.33)\end{array}$ & $\begin{array}{c}1.11 \pm 0.01^{\mathrm{f}} \\
(140.51)\end{array}$ & $\begin{array}{c}0.12 \pm 0.00^{\mathrm{c}} \\
(100)\end{array}$ & $\begin{array}{c}1.22 \pm 0.01^{*} \\
(154.43)\end{array}$ \\
\hline & KGPMF49 & $\begin{array}{l}0.03 \pm 0.00^{\mathrm{a}} \\
\quad(16.67)\end{array}$ & $\begin{array}{c}0.16 \pm 0.02^{\mathrm{b}} \\
\quad(31.37)\end{array}$ & $0.18 \pm 0.00^{\mathrm{c}}$ & $0.51 \pm 0.00^{\mathrm{d}}$ & $\begin{array}{c}0.16 \pm 0.01^{\mathrm{c}} \\
\quad(88.89)\end{array}$ & $\begin{array}{l}0.56 \pm 0.00^{\mathrm{d}} \\
\quad(109.80)\end{array}$ & $\begin{array}{l}0.15 \pm 0.00^{\mathrm{c}} \\
\quad(83.33)\end{array}$ & $\begin{array}{c}0.68 \pm 0.01^{\mathrm{e}} \\
(133.33)\end{array}$ & $\begin{array}{l}0.16 \pm 0.00^{\mathrm{c}} \\
\quad(88.89)\end{array}$ & $\begin{array}{c}0.79 \pm 0.00 \\
(154.90)\end{array}$ \\
\hline & $\begin{array}{l}\text { ATCC } \\
29211\end{array}$ & $\begin{array}{c}0.01 \pm 0.00^{\mathrm{a}} \\
(7.69)\end{array}$ & $\begin{array}{c}0.06 \pm 0.01^{\mathrm{b}} \\
(12.5)\end{array}$ & $0.13 \pm 0.01^{\mathrm{c}}$ & $0.48 \pm 0.03^{\mathrm{d}}$ & $\begin{array}{c}0.16 \pm 0.02^{\mathrm{c}} \\
(123.08)\end{array}$ & $\begin{array}{c}0.63 \pm 0.01^{\mathrm{e}} \\
(131.25)\end{array}$ & $\begin{array}{c}0.14 \pm 0.00^{c} \\
(107.69)\end{array}$ & $\begin{array}{c}0.59 \pm 0.01^{\mathrm{f}} \\
(122.92)\end{array}$ & $\begin{array}{c}0.14 \pm 0.00^{\mathrm{c}} \\
(107.69)\end{array}$ & $\begin{array}{c}0.62 \pm 0.02 \\
(129.17)\end{array}$ \\
\hline
\end{tabular}

Values are expressed as means \pm standard deviation measured on $600 \mathrm{~nm}$; the percent of growth was given in brackets.

Means in the two temperature columns or each particular isolate, with superscript with different letters, are significantly different at $P<0.05$.

*Growth control (100\% of growth). 
Table 3.

The effect of different $\mathrm{NaCl}$ concentrations and temperatures on the growth of isolated Lactobacillus spp. and Lactococcus spp.

\begin{tabular}{|c|c|c|c|c|c|c|c|}
\hline & \multirow{2}{*}{$\begin{array}{r}\text { \% of NaCl } \\
\text { Temperature }\end{array}$} & \multicolumn{2}{|c|}{4.0} & \multicolumn{2}{|c|}{6.5} & \multicolumn{2}{|c|}{8.0} \\
\hline & & $20{ }^{\circ} \mathrm{C}$ & $37^{\circ} \mathrm{C}$ & $20^{\circ} \mathrm{C}$ & $37^{\circ} \mathrm{C}$ & $20^{\circ} \mathrm{C}$ & $37^{\circ} \mathrm{C}$ \\
\hline Species & Isolate & & & & & & \\
\hline $\begin{array}{l}\text { Lb. } \\
\text { fermentum }\end{array}$ & KGPMF28 & $\begin{array}{c}0.06 \pm 0.00^{\mathrm{a}} \\
(18.18)\end{array}$ & $\begin{array}{c}0.46 \pm 0.02^{\mathrm{b}} \\
(26.44)\end{array}$ & $\begin{array}{c}0.04 \pm 0.01^{\mathrm{a}} \\
\quad(12.12)\end{array}$ & $\begin{array}{c}0.12 \pm 0.01^{\mathrm{c}} \\
\quad(24.14)\end{array}$ & n.g. & $\begin{array}{c}0.02 \pm 0.00^{\mathrm{d}} \\
(1.15)\end{array}$ \\
\hline Lb. brevis & KGPMF35 & $\begin{array}{c}0.32 \pm 0.00^{\mathrm{a}} \\
(74.42)\end{array}$ & $\begin{array}{c}1.62 \pm 0.01^{\mathrm{b}} \\
(78.26)\end{array}$ & $\begin{array}{c}0.12 \pm 0.01^{\mathrm{c}} \\
(27.91)\end{array}$ & $\begin{array}{c}0.22 \pm 0.02^{\mathrm{d}} \\
(10.63)\end{array}$ & n.g. & $\begin{array}{c}0.09 \pm 0.01^{\mathrm{e}} \\
(4.35)\end{array}$ \\
\hline \multirow{2}{*}{$\begin{array}{l}\text { Lb. } \\
\text { plantarum }\end{array}$} & KGPMF62 & $\begin{array}{l}0.32 \pm 0.02^{\mathrm{a}} \\
\quad(72.72)\end{array}$ & $\begin{array}{c}1.70 \pm 0.02^{\mathrm{b}} \\
(83.33)\end{array}$ & $\begin{array}{l}0.09 \pm 0.00^{c} \\
\quad(20.45)\end{array}$ & $\begin{array}{c}0.13 \pm 0.01^{c, d} \\
(6.37)\end{array}$ & n.g. & $\begin{array}{c}0.10 \pm 0.00^{\mathrm{d}} \\
(4.91)\end{array}$ \\
\hline & LP 299v & $\begin{array}{c}0.19 \pm 0.01^{\mathrm{a}} \\
(65.52)\end{array}$ & $\begin{array}{c}0.45 \pm 0.01^{\mathrm{b}} \\
(91.84)\end{array}$ & $\begin{array}{c}0.08 \pm 0.00^{\mathrm{c}} \\
(27.59)\end{array}$ & $\begin{array}{c}0.17 \pm 0.01^{\mathrm{d}} \\
(34.69)\end{array}$ & n.g. & $\begin{array}{c}0.03 \pm 0.00^{\mathrm{e}} \\
(6.12)\end{array}$ \\
\hline \multirow{5}{*}{$\begin{array}{l}\text { L. lactis } \\
\text { subsp. lactis } \\
\text { biovar. } \\
\text { diacetylactis }\end{array}$} & KGPMF50 & $\begin{array}{c}0.10 \pm 0.00^{\mathrm{a}} \\
(43.49)\end{array}$ & $\begin{array}{c}0.61 \pm 0.01^{\mathrm{b}} \\
(52.14)\end{array}$ & $\begin{array}{c}0.05 \pm 0.01^{\mathrm{c}} \\
(21.74)\end{array}$ & $\begin{array}{c}0.22 \pm 0.01^{\mathrm{d}} \\
(18.80)\end{array}$ & $\begin{array}{c}0.02 \pm 0.0^{\mathrm{e}, \mathrm{c}} \\
(8.70)\end{array}$ & $\begin{array}{c}0.05 \pm 0.01^{\mathrm{f}} \\
(4.27)\end{array}$ \\
\hline & KGPMF54 & $\begin{array}{c}0.08 \pm 0.01^{\mathrm{a}} \\
\quad(47.06)\end{array}$ & $\begin{array}{c}1.42 \pm 0.01^{\mathrm{b}} \\
(70.30)\end{array}$ & $\begin{array}{c}0.04 \pm 0.01^{\mathrm{c}} \\
\quad(23.53)\end{array}$ & $\begin{array}{c}0.11 \pm 0.00^{\mathrm{d}} \\
\quad(5.45)\end{array}$ & $\begin{array}{c}0.03 \pm 0.00^{\mathrm{c}} \\
\quad(17.65)\end{array}$ & $\begin{array}{c}0.09 \pm 0.00^{\mathrm{d}} \\
\quad(4.46)\end{array}$ \\
\hline & KGPMF55 & $\begin{array}{c}0.05 \pm 0.01^{\mathrm{a}} \\
\quad(31.25)\end{array}$ & $\begin{array}{c}0.78 \pm 0.01^{\mathrm{b}} \\
\quad(45.09)\end{array}$ & $\begin{array}{c}0.02 \pm 0.00^{\mathrm{a}} \\
\quad(12.5)\end{array}$ & $\begin{array}{c}0.20 \pm 0.01^{\mathrm{c}} \\
\quad(11.56)\end{array}$ & $\begin{array}{l}0.01 \pm 0.00^{\mathrm{a}} \\
\quad(6.25)\end{array}$ & $\begin{array}{c}0.03 \pm 0.00^{\mathrm{d}} \\
\quad(1.73)\end{array}$ \\
\hline & KGPMF57 & $\begin{array}{c}0.12 \pm 0.02^{\mathrm{a}} \\
\quad(54.55)\end{array}$ & $\begin{array}{c}1.69 \pm 0.02^{\mathrm{b}} \\
(82.84)\end{array}$ & $\begin{array}{c}0.05 \pm 0.01^{\mathrm{c}} \\
(22.73)\end{array}$ & $\begin{array}{c}0.13 \pm 0.00^{\mathrm{d}} \\
\quad(6.37)\end{array}$ & $\begin{array}{c}0.02 \pm 0.00^{\mathrm{c}} \\
(9.09)\end{array}$ & $\begin{array}{c}0.10 \pm 0.00^{\mathrm{d}} \\
\quad(4.90)\end{array}$ \\
\hline & KGPMF59 & $\begin{array}{c}0.14 \pm 0.02^{\mathrm{a}} \\
\quad(53.85)\end{array}$ & $\begin{array}{c}1.62 \pm 0.02^{\mathrm{b}} \\
(79.41)\end{array}$ & $\begin{array}{c}0.12 \pm 0.00^{\mathrm{a}} \\
\quad(46.15)\end{array}$ & $\begin{array}{c}0.16 \pm 0.00^{c} \\
(7.84)\end{array}$ & $\begin{array}{c}0.03 \pm 0.00^{\mathrm{d}} \\
(11.54)\end{array}$ & $\begin{array}{c}0.12 \pm 0.00^{\mathrm{c}} \\
\quad(5.88)\end{array}$ \\
\hline
\end{tabular}

Values are expressed as means \pm standard deviation measured on $600 \mathrm{~nm}$; the percent of growth was given in brackets; Means in the two temperature columns or each particular isolate, with superscript with different letters, are significantly different at $P<0.05 ;$ n.g.-no growth.

Table 4.

The effect of different $\mathrm{NaCl}$ concentrations and temperatures on the growth of isolated Enterococcus spp.

\begin{tabular}{|c|c|c|c|c|c|c|c|}
\hline & \multirow{2}{*}{$\begin{array}{r}\% \text { of } \mathrm{NaCl} \\
\text { Temperature }\end{array}$} & \multicolumn{2}{|c|}{4.0} & \multicolumn{2}{|c|}{6.5} & \multicolumn{2}{|c|}{8.0} \\
\hline & & $20{ }^{\circ} \mathrm{C}$ & $37^{\circ} \mathrm{C}$ & $20{ }^{\circ} \mathrm{C}$ & $37^{\circ} \mathrm{C}$ & $20^{\circ} \mathrm{C}$ & $37^{\circ} \mathrm{C}$ \\
\hline Species & Isolate & & & & & & \\
\hline E. durans & KGPMF10 & $\begin{array}{c}0.32 \pm 0.01^{\mathrm{a}} \\
(50)\end{array}$ & $\begin{array}{c}0.97 \pm 0.03^{\mathrm{b}} \\
(75.40)\end{array}$ & $\begin{array}{c}0.11 \pm 0.01^{\mathrm{c}} \\
(17.19)\end{array}$ & $\begin{array}{c}0.22 \pm 0.00^{\mathrm{d}} \\
(13.02)\end{array}$ & $\begin{array}{c}0.02 \pm 0.00^{\mathrm{e}} \\
\quad(3.13)\end{array}$ & $\begin{array}{c}0.08 \pm 0.01^{\mathrm{f}} \\
(4.73)\end{array}$ \\
\hline E. faecium & KGPMF14 & $\begin{array}{c}0.06 \pm 0.01^{\mathrm{a}, \mathrm{f}} \\
(66.67)\end{array}$ & $\begin{array}{c}0.45 \pm 0.01^{\mathrm{b}} \\
(91.84)\end{array}$ & $\begin{array}{c}0.03 \pm 0.00^{\mathrm{a}} \\
(33.33)\end{array}$ & $\begin{array}{c}0.10 \pm 0.01^{\mathrm{c}} \\
(20.41)\end{array}$ & n.g. & $\begin{array}{c}0.03 \pm 0.00^{\mathrm{d}} \\
(6.12)\end{array}$ \\
\hline \multirow{4}{*}{ E. faecalis } & KGPMF47 & $\begin{array}{c}0.03 \pm 0.00^{\mathrm{a}} \\
(37.5)\end{array}$ & $\begin{array}{c}0.40 \pm 0.00^{\mathrm{b}} \\
(83.33)\end{array}$ & $\begin{array}{l}0.01 \pm 0.0^{\mathrm{a}} \\
(12.5)\end{array}$ & $\begin{array}{c}0.07 \pm 0.00^{\mathrm{c}} \\
(14.58)\end{array}$ & n.g. & $\begin{array}{c}0.03 \pm 0.00^{\mathrm{d}} \\
(6.25)\end{array}$ \\
\hline & KGPMF48 & $\begin{array}{c}0.09 \pm 0.00^{\mathrm{a}} \\
\quad(75)\end{array}$ & $\begin{array}{c}0.31 \pm 0.11^{\mathrm{b}} \\
\quad(39.24)\end{array}$ & $\begin{array}{c}0.06 \pm 0.00^{\mathrm{a}} \\
(50)\end{array}$ & $\begin{array}{c}0.10 \pm 0.01^{\mathrm{c}} \\
(12.66)\end{array}$ & $\begin{array}{c}0.02 \pm 0.00^{\mathrm{a}} \\
\quad(16.67)\end{array}$ & $\begin{array}{c}0.03 \pm 0.01^{\mathrm{d}} \\
(3.80)\end{array}$ \\
\hline & KGPMF49 & $\begin{array}{c}0.12 \pm 0.00^{\mathrm{a}} \\
\quad(66.67)\end{array}$ & $\begin{array}{c}0.43 \pm 0.01^{\mathrm{b}} \\
(84.31)\end{array}$ & $\begin{array}{l}0.03 \pm 0.00^{\mathrm{c}} \\
\quad(16.67)\end{array}$ & $\begin{array}{c}0.08 \pm 0.00^{\mathrm{d}} \\
(15.69)\end{array}$ & n.g. & $\begin{array}{c}0.03 \pm 0.00^{\mathrm{e}} \\
(5.88)\end{array}$ \\
\hline & ATCC 29211 & $\begin{array}{c}0.08 \pm 0.01^{\mathrm{a}} \\
\quad(61.54)\end{array}$ & $\begin{array}{c}0.43 \pm 0.01^{\mathrm{b}} \\
(89.58)\end{array}$ & $\begin{array}{c}0.03 \pm 0.00^{\mathrm{c}} \\
(23.08)\end{array}$ & $\begin{array}{c}0.09 \pm 0.01^{\mathrm{d}} \\
(18.75)\end{array}$ & $\begin{array}{c}0.01 \pm 0.00^{\mathrm{c}} \\
(2.08)\end{array}$ & $\begin{array}{c}0.03 \pm 0.00^{\mathrm{e}} \\
(6.25)\end{array}$ \\
\hline
\end{tabular}

Values are expressed as means \pm standard deviation measured on $600 \mathrm{~nm}$; the percent of growth was given in brackets; Means in the two temperature columns or each particular isolate, with superscript with different letters, are significantly different at $P<0.05$; n.g.-no growth.

Previously, isolates of LAB showed a good acidification activity in original and enriched milk (Muruzović, Mladenović, Žugić Petrović \& Čomić, 2018a; Muruzović, Mladenović \& Čomić, 2018b; Grujović, Mladenović, Žugić Petrović \& Čomić, 2019). We selected isolates that showed the ability to decrease $\mathrm{pH}$ value to almost 4 within $24 \mathrm{~h}$ because this was a promising result which indicated the ability to use the selected isolate as starter cultures for food fermentations. They showed the ability to consume sugars from the food matrix converting them to acid via the fast fermentation process. Such produced acids are also attractive criteria for selected strains to be protective cultures where pathogenic bacteria cannot grow, due to a low $\mathrm{pH}$ value of the medium.

Some of the research increasingly suggests that the acid resistance of the LAB might be strainspecific and stress-specific. The reason can be found in the genetic diversity of these acid alleviating systems among different strains (Wu, Tun, Law, Khafipour \& Shah, 2017; Lyu et al., 2018). Lb. fermentum KGPMF29, isolated from Sokobanja cheese, was able to to- 
lerate the acidic $\mathrm{pH}$ (Muruzović, Mladenović \& Čomić, 2018b). Rao, Pintado, Stevens \& Guyot (2004) and Muruzović, Mladenović \& Čomić (2018b) indicated that Lb. fermentum was not tolerant to higher $\mathrm{NaCl}$ concentrations. Soliman, Sharoba, Bahlol, Soliman \& Radi (2015) showed that $L b$. plantarum had the ability of tolerance to acidic medium ( $\mathrm{pH} 2.0$ and 3.0) and bile salts $(0.1,0.3,0.5$, and $0.7 \%)$, at $37^{\circ} \mathrm{C}$ for $24 \mathrm{~h}$. Lb. plantarum ATCC 14917 showed the ability of growth in $6 \%$ of $\mathrm{NaCl}$ (Wang et al., 2016), while $L b$. plantarum B282 showed a survival rate in $8 \%$ of $\mathrm{NaCl}$ (Blana, Grounta, Tassou, Nychas \& Panagou, 2014), which was confirmed in our study, too. Xia et al. (2017) indicated that optimal $\mathrm{NaCl}$ concentration in the medium is $6 \%$ because in this concentration Lb. brevis AR123 and a commercial starter could rapidly lower the $\mathrm{pH}$ of the pickles. The results from our investigation confirmed that tested Lactobacillus isolates were more tolerant to acidic $\mathrm{pH}$, but their tolerance to the presence of $\mathrm{NaCl}$ concentration higher than $6.5 \%$ was low.

Lactococcus lactis subsp. lactis is one of the most important starter bacteria used in dairy technology. Its use in the production of dairy products, such as cheese, butter, cream, and fermented kinds of milk is of great economic importance (Yerlikaya, 2019). It is well-known that a starter culture bacterium, L. lactis needs to be tolerant to a variety of processing conditions (high or low temperature, osmotic and acid environment) in the process of cheese making (Gutiérrez-Méndez et al., 2010). Velly, Fonseca, Passot, Delacroix-Buchet \& Bouix (2014) investigated the effects of fermentation parameters (different temperatures $\left(22{ }^{\circ} \mathrm{C}, 30\right.$ ${ }^{\circ} \mathrm{C}$ and $\left.38{ }^{\circ} \mathrm{C}\right)$ and $\mathrm{pH}(5.6,6.2$ and 6.8$\left.)\right)$ on the cell growth and on the tolerance to each step of the freeze-drying process of natural cheese isolate L. lactis subsp. lactis TOMSC161. They concluded that, in the whey-based medium, $L$. lactis showed the best growth at $32{ }^{\circ} \mathrm{C}, \mathrm{pH}$ 6.2. Khemariya, Singh, Nath \& Gulati (2017) indicated that the growth of Lactococcus spp. at higher $\mathrm{NaCl}$ concentrations (>4\% sodium chloride) vary depending on the species. Muruzović, Mladenović \& Čomić (2018b) showed that L. lactis subsp. lactis KGPMF23 showed the ability of low growth in $6.5 \%$ of $\mathrm{NaCl}$, at $37{ }^{\circ} \mathrm{C}$. The results from this paper indicated that tested Lactococcus isolates were tolerant to acidic $\mathrm{pH}$, as well as in the different $\mathrm{NaCl}$ concentrations, but in concentrations above $6.5 \%$, the growth was reduced. Van Mastrigt, Gallegos Tejeda, Kristensen, Abee \& Smid (2018) indicated that aroma production by L. lactis was clearly affected by the type of medium and the cultivation method. Therefore, the optimal conditions for the growth of bacteria might be strain-specific and stressspecific.

Enterococci can grow in a wide range of $\mathrm{pH}$, but the optimum for growth is $\mathrm{pH} 7.5$ (Van Den Berghe, De Winter \& De Vuyst, 2006). Fisher \& Phillips (2009) also indicated that E. faecalis showed the ability of growth in $6.5 \%$ of $\mathrm{NaCl}$. Some authors indicated that E. durans, E. hirae, and $E$. faecium strains isolated from raw milk and various dairy products were resistant to low $\mathrm{pH}$ and to the different bile $\mathrm{NaCl}$ concentrations (Nami et al.,2014; Guo, Li, Tang, Yang \& Huo, 2016; Muruzović, Mladenović, \& Čomić 2018b; Yerlikaya \& Akbulut, 2020). Ivanov, Boytcheva \& Mihailova (1999) indicated that temperature influenced E. faecalis membrane permeability for salts. In our study, tested Enterococcus isolates showed a high tolerance in the basic $\mathrm{pH}$ and presence of $\mathrm{NaCl}$. Although the role of bacteria from genus Enterococcus in the formation of cheese flavor is proved (Abeijón, Medina, Katz \& Gonzalez, 2006), a very few Enterococcus strains have been used as food additives because of the safety concern associated with their pathogenic trait as opportunistic microorganisms (Braiek \& Smaoui, 2019).

In most cases, traditionally made dairy products are produced by spontaneous milk fermentation, without the addition of any bacterial starter culture. Autochthonous lactic acid bacteria $(\mathrm{aLAB})$, that are present in milk, are responsible for fermentation and quality of dairy products. Most of the research conducted so far confirmed that aLAB has great potential in natural food preservation and good probiotic properties. There are studies which indicated that the adding of natural isolates, with a desirable probiotic's characteristic, in the production of the dairy product have a positive effect on the sensory properties, especially on flavor (Silva et al., 2017: Baher Abd EL Khalek et al., 2018; Santos et al., 2018; Yerlikaya \& Akbulut, 2019). 


\section{CONCLUSIONS}

The tested processing conditions (different temperatures, $\mathrm{pH}$, and $\mathrm{NaCl}$ concentrations) showed a different influence on the growth of the tested LAB. After $24 \mathrm{~h}$ of incubation, growth at $4{ }^{\circ} \mathrm{C}$ was not noticed. Generally, all tested isolates showed the ability of growth in low $\mathrm{pH}$ (acidic media), as well as up to $6.5 \%$ of $\mathrm{NaCl}$, which is a desirable characteristic for potential starter culture. This paper indicated that the tests showed certain positive technological properties of the tested LAB isolates. This is an important issue because if we use these bacteria like starter cultures, the safety aspect and hygiene of cheese will be increased together with the conservation of traditional heritage, while the aroma and taste will be preserved. Further studies need to include the characterrization of enzymatic activities and amine formation, the effect on texture and organoleptic properties of products of selected isolates, in order to investigate their role in the formation of cheese flavor and taste. Also, Enterococcus strains investigated for the potential use must be well characterized and perfectly assessed regarding safety aspects.

\section{ACKNOWLEDGEMENTS}

This investigation was supported by the Ministry of Education, Science and Technological Development of the Republic of Serbia (Agreement No. 451-03-68/2020-14/200122).

\section{REFERENCES}

Abeijón, M. C., Medina, R. B., Katz, M. B., \& Gonzalez, S. N. (2006). Technological properties of Enterococcus faecium isolated from ewe's milk and cheese with importance for flavour development. Canadian Journal of Microbiology, 52(3), 237-245. https://doi.org/10.1139/w05-136

Adamberg, K., Kask, S., Laht, T.M., \& Paalme, T. (2003). The effect of temperature and $\mathrm{pH}$ on the growth of lactic acid bacteria: A pH-auxostat study. International Journal of Food Microbiology, 85, 171-183. https://doi.org/10.1016/S0168-1605(02)00537-8

Baher Abd El Khalek, M. E., Hassan, Z. M. R., Mabrouk, A. M. M., Sadek, Z. I. M., Magdoub, M. N. I., \& Tawfik, N. F. (2018). Properties of low salt soft cheese supplemented with probiotic cultures. International Journal of Advanced Research in Biological Science, 5, 1-10. http://dx.doi.org/10.22192/ijarbs.2018.05.02.001

Blana, V. A., Grounta, A., Tassou, C. C., Nychas, G. J. E., \& Panagou, E. Z. (2014). Inoculated fermentation of green olives with potential probiotic Lactobacillus pentosus and Lactobacillus plantarum starter cultures isolated from industrially fermented olives. Food Microbiology, 38, 208-218. https://doi.org/10.1016/j.fm.2013.09.007

Braiek, O. B., \& Smaoui, S. (2019). Enterococci: between emerging pathogens and potential probiotics. BioMed Research International, 2019, 1 - 13. https://doi.org/10.1155/2019/5938210

Fisher, K., \& Phillips, C. (2009). The ecology, epidemiology and virulence of Enterococcus. Microbiology, 155,1749-1757. https://doi.org/10.1099/mic.0.026385-0

Fontana, L., Bermudez-Brito, M., Plaza-Diaz, J., MuñozQuezada, S., \& Gil, A. (2013). Sources, isolation, characterisation and evaluation of probiotics. British Journal of Nutrition, 109, 35-50. https://doi.org/10.1017/S0007114512004011

Fox, P. F., Guinee, T. P., Cogan, T. M., \& McSweeney, P. L. H. (2017). Biochemistry of cheese ripening. In P. F. Fox, T. P. Guinee, T. M. Cogan, \& P. L. H. McSweeney (Eds.), Fundamentals of cheese science (pp. 391-44). Boston: Springer.

Grujović, M., Mladenović, K., Žugić Petrović, T., \& Comić, L. (2019). Assessment of the antagonistic potential and ability of biofilm formation of Enterococcus spp. isolated from Serbian cheese. Veterinarski arhiv, 89, 653-667. https://doi.org/10.24099/vet.arhiv.0485

Guo, L., Li, T., Tang, Y., Yang, L., \& Huo, G. (2016). Probiotic properties of Enterococcus strains isolated from traditional naturally fermented cream in China. Microbial Biotechnology, 9, 737-745. https://doi.org/10.1111/1751-7915.12306

Gutiérrez-Méndez, N., Rodríguez-Figueroa, J. C., González-Cordova, A. F., Nevárez-Moorillón, G. V., Rivera-Chavira, B., \& Vallejo-Cordoba, B. (2010). Phenotypic and genotypic characteristics of Lactococcus lactis strains isolated from different ecosystems. Canadian Journal of Microbiology, 56, 432-439. https://doi.org/10.1139/W10-026

Hernandez-Hernandez, O. A., Muthaiyan, F. J., Moreno, A., Montilla, M. L., Sanz, S., \& Rickeet, C. (2012). Effect of prebiotic carbohydrates on the growth and tolerance of Lactobacillus. Food Microbiology, 30, 355-361. https://doi.org/10.1016/j.fm.2011.12.022

Ibourahema, C., Dauphin, R. D., Jacqueline, D., \& Thonart, P. (2008). Characterization of lactic acid bacteria isolated from poultry farms in Senegal. African Journal of Biotechnology, 7, 2006-2012. https://doi.org/10.5897/AJB2008.000-5048

Ivanov, I. T., Boytcheva, S., \& Mihailova, G. (1999). Parallel study of thermal resistance and permeability barrier stability of Enterococcus faecalis as affected by salt composition, growth temperature and pre-incubation temperature. Journal of Thermal Biology, 24,217-227.

https://doi.org/10.1016/S0306-4565(99)00012-1

Kavitha, J. R., \& Devasena, T. (2013). Isolation, characterization, determination of probiotic properties of lactic acid bacteria from human milk. Journal of Pharmaceutical and Biological Sciences, 7, 1-7. https://doi.org/10.9790/3008-0730107

Khemariya, P., Singh, S., Nath, G., \& Gulati, A. K. (2017). Probiotic Lactococcus lactis: A review. Turkish Journal of Agriculture - Food Science and Technology, 5, 556-562. https://doi.org/10.24925/turjaf.v5i6.556-562.690

Lyu, C., Zhao, W., Peng, C., Hu, S., Fang, H., Hua, Y., Yao, S., Huang, J., \& Mei, L. (2018). Exploring the 
contributions of two glutamate decarboxylase isozymes in Lactobacillus brevis to acid resistance and $\gamma$-aminobutyric acid production. Microbial Cell Factories, 17,180. https://doi.org/10.1186/s12934018-1029-1

Menconi, A., Kallapura, G., Latorre, J. D., Morgan, M. J., Pumford, N. R., Hargis, B. M., \& Tellez, G. (2014). Identification and characterization of lactic acid bacteria in a commercial probiotic culture. Bioscience of Microbiota, Food and Health, 33, 25-30. https://doi.org/10.12938/bmfh.33.25

Mohd Adnan, A. F., \& Tan, I. K. (2007). Isolation of lactic acid bacteria from Malaysian foods and assessment of the isolates for industrial potential. Bioresource Technology, 98, 1380-1385. https://doi.org/10.1016/j.biortech.2006.05.034

Muruzović, M., Mladenović, K., Žugić Petrović, T., \& Čmić, L. (2018a). Characterization of lactic acid bacteria isolated from traditionally made Serbian cheese and evaluation of their antagonistic potential against Enterobacteriaceae. Journal of Food Processing and Preservation, 42, e13577. https://doi.org/10.1111/jfpp.13577

Muruzović, M., Mladenović, K., \& Čomić, L. (2018b). In vitro evaluation of resistance to environmental stress by planktonic and biofilm form of lactic acid bacteria isolated from traditionally made cheese from Serbia. Food Bioscience, 23, 54-59. https://doi.org/10.1016/j.fbio.2018.03.005

Muruzović, M., Mladenović, K., Đilas, M., Stefanović, O., \& Čomić, L. (2018c). In vitro evaluation of antimicrobial potential and ability of biofilm formation of autochthonous Lactobacillus spp. and Lactococcus spp. isolated from traditionally made cheese from Southeastern Serbia. Journal of Food Processing and Preservation, 42(11), e13776. https://doi.org/ 10.1111/jfpp.13776

Nami, Y., Abdullah, N., Haghshenas, B., Radiah, D., Rosli, R., \& Khosroushahi, A. Y. (2014). Probiotic assessment of Enterococcus durans 6HL and Lactococcus lactis 2HL isolated from vaginal microflora. Journal of Medical Microbiology, 63, 1044-1051. https://doi.org/10.1099/jmm.0.0741610

Rao, M. S., Pintado, J., Stevens, W. F., \& Guyot, J. P. (2004). Kinetic growth parameters of different amylolytic and non-amylolytic Lactobacillus strains under various salt and $\mathrm{pH}$ conditions. Bioresource Technology, 94, 331-337. https://doi.org/10.1016/j.biortech.2003.11.028

Santos, R. O., Silva, M. V. F., Nascimento, K. O., Batista, A. L. D., Moraes, J., Andrade, M. M., Andrade, L. G. Z. S., Khosravi-Darani, K., Freitas, M. Q., Raices, R. S. L., Silva, M. C., Barbosa Junior, J. L., Barbosa, M. I. M. J., \& Cruz, A. G. (2018). Prebiotic flours in dairy food processing: Technological and sensory implications. International Journal of Dairy Technology, 70, 1-10. https://doi.org/10.1111/1471-0307.12394

Silva, H. L. A., Balthazar, C. F., Esmerino, E. A., Vieira, A. H., Cappato, L. P., Neto, R. P. C., Verruck, S., Cavalcanti, R. N., Portela, J. B., Andrade, M. M., Moraes, J., Franco, R. M., Tavares, M. I. B., Prudencio, E. S., Freitas, M. Q., Nascimento, J. S., Silva, M. C., Raices, R. S. L., \& Cruz, A. (2017). Effect of sodium reduction and flavor enhancer addition on probiotic Prato cheese processing. Food Research International, 99, 247-255.

https://doi.org/10.1016/j.foodres.2017.05.018

Soliman, A. H. S., Sharoba, A. M., Bahlol, H. E. M., Soliman, A. S., \& Radi, O. M. M. (2015). Evaluation of Lactobacillus acidophilus, Lactobacillus casei and Lactobacillus plantarum for probiotic characteristics. Middle East Journal of Applied Science, 5, 10-18.

Thayer, D. W., Muller, W. S., Buchanan, R. L., \& Phillips, J. G. (1987). Effect of $\mathrm{NaCl}, \mathrm{pH}$, temperature and atmosphere on growth of Salmonella typhimurium in glucose-mineral salts medium. Applied and Environmental Microbiology, 53, 1311-1315. https://doi.org/10.1128/AEM.53.6.1311-1315.1987

Uroić, K., Nikolić, M., Koslć, B., Pavunc, L., Beganović, J., Lukić, J., Jovčić, B., Filipić, B., Miljković, M., Golić, N., Topisirović, L., Čadež, N., Raspor, P., \& Šušković, J. (2014). Probiotic properties of lactic acid bacteria isolated from Croatian fresh soft cheese and Serbian white pickled cheese. Food Technology and Biotechnology, 52, 232-241.

Van Den Berghe, E., De Winter, T., \& De Vuyst, L. (2006). Enterocin A production by Enterococcus faecium FAIR-E 406 is characterised by a temperature and $\mathrm{pH}$-dependent switch-off mechanism when growth is limited due to nutrient depletion. International Journal of Food Microbiology, 107, 159-170. https://doi.org/10.1016/j.ijfoodmicro.2005.08.027

Van Mastrigt, O., Gallegos Tejeda, D., Kristensen, M. N., Abee, T., \& Smid, E. J. (2018). Aroma formation during cheese ripening is best resembled by Lactococcus lactis retentostat cultures. Microbial Cell Factories, 17, 104.

https://doi.org/10.1186/s12934-018-0950-7

Velly, H., Fonseca, F., Passot, S., Delacroix-Buchet, A., \& Bouix, M. (2014). Cell growth and resistance of Lactococcus lactis subsp. lactis TOMSC161 following freezing, drying and freeze-dried storage are differentially affected by fermentation conditions. Journal of Applied Microbiology, 117, 729740. https://doi.org/10.1111/jam.12577

Wang, P., Wu, Z., Wu, J., Pan, D., Zen, X., \& Cheng. (2016). Effects of salt stress on carbohydrate metabolism of Lactobacillus plantarum ATCC 14917. Current Microbiology, 73, 491-497. https://doi.org/10.1007/s00284-016-1087-8

Wu, Q., Tun, H. M., Law, Y. S., Khafipour, E., \& Shah, N. P. (2017). Common distribution of gad operon in Lactobacillus brevis and its GadA contributes to efficient GABA synthesis toward cytosolic nearneutral pH. Frontiers in Microbiology, 8, 206. https://doi.org/10.3389/fmicb.2017.00206

Xia, Y., Liu, X., Wang, G., Zhang, H., Xionga, Z., Sun, Y., \& Ai, L. (2017). Characterization and selection of Lactobacillus brevis starter for nitrite degradation of Chinese pickle. Food Control, 78, 126131. https://doi.org/10.1016/j.foodcont.2017.02.046

Yerlikaya, O. (2019). Probiotic potential and biochemical and technological properties of Lactococcus lactis ssp. lactis strains isolated from raw milk and kefir grains. Journal of Dairy Science, 102, 124-134. https://doi.org/10.3168/jds.2018-14983

Yerlikaya, O., \& Akbulut, N. (2019). Potential use of probiotic Enterococcus faecium and Enterococcus 
durans strains in Izmir Tulum cheese as adjunct culture. Journal of Food Science and Technology Mysore, 56, 2175-2185.

https://doi.org/10.1007/s13197-019-03699-5

Yerlikaya, O., \& Akbulut, N. (2020). In vitro characterisation of probiotic properties of Enterococcus faecium and Enterococcus durans strains isolated from raw milk and traditional dairy products. Journal of Dairy Technology, 73, 98-107. https://doi.org/10.1111/1471-0307.12645

Zago, M., Fornasari, M. E., Carminati, D., Burns, P., Suàrez, V., Vinderola, G., Reinheimer, J., \& Giraffa, G. (2011). Characterization and probiotic potential of Lactobacillus plantarum strains isolated from cheeses. Food Microbiology, 28, 1033-1040.

https://doi.org/10.1016/j.fm.2011.02.009 


\title{
ТОЛЕРАНЦИЈА АУТОХТОНИХ БАКТЕРИЈА МЛЕЧНЕ КИСЕЛИНЕ НА РАЗЛИЧИТЕ УСЛОВЕ
} ПРЕРАДЕ IN VITRO

\author{
Мирјана Ж. Грујовић, Катарина Г. Младеновић, Љиљана Р. Чомић
}

Универзитет у Крагујевцу, Природно-математички факултет, Институт за биологију и екологију, Радоја Домановића 12, 34000 Крагујевац, Србија

Сажетак: У овом раду је испитиван утицај различитих температура, $\mathrm{pH}$ и концентрација соли на раст аутохтоних бактерија млечне киселине изолованих из традиционално произведеног српског сира (подручје Сокобање) помоћу спектрофотометријске методе. Раст испитиваних Lactobacillus изолата (Lb. fermentum, Lb. plantarum и Lb. brevis), као и пет изолата Lactococcus lactis subsp. lactis biovar. diacetylactis је био бољи у киселој $\mathrm{pH}$, док је раст изолата Enterococcus (E. durans, E. faecium и E. faecalis) био бољи у базној pH, на $37{ }^{\circ} \mathrm{C}$. На $4{ }^{\circ} \mathrm{C}$ након 24 часа, ниједна тестирана бактерија није показала способност раста. Будући да су аутохтони изолати били толерантни на тестирани спектар услова прераде млека, даље студије морају да обухвате карактеризацију ензимске активности одабраних изолата, као и могућност употребе ових изолата као стартер култура или додатака исхрани у млечним или немлечним производима.

Кључне речи: бактерије млечне киселине, микробиологија сира, услови прераде, способност раста

Received: 26 August 2020 / Received in revised form: 27 October 2020 / Accepted: 03 November 2020

Available online: November 2020

This is an open-access article under the CC BY license (http://creativecommons.org/licenses/by/3.0). 